\title{
Los motivos del aprendizaje en la primaria desde la teoría histórico-cultural
}

\author{
The motives for learning in primary school from \\ historical-cultural theory
}

Adriana Mata-Esquivel ${ }^{1}$

\begin{abstract}
RESUMEN
En este artículo se presenta la aproximación hacia la comprensión de los motivos relacionados con el aprendizaje escolar en niños de primaria desde el enfoque histórico-cultural y la teoría de la actividad aplicada a la enseñanza. Esta aproximación representa una nueva concepción para el estudio de la motivación escolar, alejada de la visión predominante que limita la motivación escolar a los aspectos afectivosemocionales. En este enfoque los motivos constituyen la representación interna (mental) de los objetivos que cubren las diferentes actividades del hombre incluyendo la actividad escolar (VIGOTSKY, 1996a, TALIZINA, 1998). Leontiev (1993) desarrollo la Teoría de la actividad general a partir de las ideas de Vigotsky sobre la naturaleza social de la psique, la cual se manifiesta y desarrolla en la actividad. El autor señaló que la actividad del ser humano en general y la actividad escolar en particular deben ser estudiadas desde el aspecto motivacional para entender la relación entre las diferentes necesidades y los objetivos que las satisfacen. En la teoría de la actividad se señala que la efectividad o fracaso en el proceso de enseñanza-aprendizaje se relaciona directamente con el tipo de motivos, los cuales forman los sentidos de la actividad escolar en los niños (BOZHOVICH, 1987; LEONTIEV, 1993; TALIZINA, 2009; VIGOTSKY, 1996a, 1996b).
\end{abstract}

Palavras-chave: Motivación escolar. Desmotivación. Motivos del aprendizaje.

\begin{abstract}
This article presents an approach for understanding the motives related to school learning in elementary school children from the historical-cultural approach and the theory of activity applied to teaching. This approach represents a new conception for the school motivation study, away from the predominant vision that limits school motivation to affective-emotional aspects. In this approach, the motives constitute the internal (mental) representation of the objectives that cover the different activities of man, including school activity (VIGOTSKY, 1996a, TALIZINA, 1998). Leontiev (1993) developed the General Activity Theory from Vygotsky's ideas about the social nature of the psyche, which manifests and develops in activity. The author pointed out that human activity in general and school activity in particular must be studied from the motivational aspect in order to understand the relationship between different needs and the objectives that satisfy them. In the activity theory it is pointed out that the effectiveness or failure in the teachinglearning process is directly related to the type of motives, which forms the meanings of school activity in children (BOZHOVICH, 1987; LEONTIEV, 1993; TALIZINA, 2009; VIGOTSKY, 1996a, 1996b).
\end{abstract}

Keywords: School motivation. Demotivation. Learning motives.

\footnotetext{
${ }^{1}$ Doctorado Interinstitucional en Educación, Ibero-Puebla. Master en Rehabilitación Neurológica, UAM-México. Lic. Psicología FESZ-UNAM. Directora del Instituto de Neuropsicología (INPIMéxico. ORCID: https://orcid.org/0000-0003-1534-4984. E-mail: adrianamata5@hotmail.com.
} 


\section{RESUMO}

Este artigo apresenta uma abordagem para a compreensão dos motivos relacionados à aprendizagem escolar em crianças do ensino fundamental a partir da abordagem histórico-cultural e da teoria da atividade aplicada ao ensino. Essa abordagem representa uma nova concepção para o estudo da motivação escolar, afastando-se da visão predominante que limita a motivação escolar aos aspectos afetivo-emocionais. Nessa abordagem, os motivos constituem a representação interna (mental) dos objetivos que abrangem as diferentes atividades do homem, incluindo a atividade escolar (VIGOTSKY, 1996a, TALIZINA, 1998). Leontiev (1993) desenvolveu a Teoria da Atividade a partir das ideias de Vygotsky sobre a natureza social da psique, que se manifesta e se desenvolve no atividade.
$\mathrm{O}$ autor destacou que a atividade humana em geral e a atividade escolar em particular devem ser estudadas do ponto de vista motivacional para compreender a relação entre as diferentes necessidades e os objetivos que as satisfazem. $\mathrm{Na}$ teoria da atividade aponta-se que a eficácia ou fracasso no processo de ensino-aprendizagem está diretamente relacionada ao tipo de motivos que constituem os significados da atividade escolar nas crianças (BOZHOVICH, 1987; LEONTIEV, 1993; TALIZINA, 2009; VIGOTSKY, 1996a, 1996b

Palavras-chave: Motivação escolar. Desmotivação. Motivos de aprendizagem.

\section{Introducción}

En la última década los resultados de los estudios internacionales para mejorar la calidad de la educación han alentado la investigación sobre la motivación escolar. La Organización para la Cooperación y el Desarrollo Económicos (OCDE) (2016) reconoce a la motivación hacia el estudio como un aspecto estrechamente relacionado con el éxito o fracaso escolar. Desde la primera edición del Programa Internacional de Evaluación de Estudiantes (PISA) del año 2000 (OCDE, 2002), que se aplica a los alumnos que finalizan su etapa secundaria, se señala en los informes que tanto la motivación como la dedicación son elementos fundamentales para el aprendizaje permanente, son factores que posibilitan la realización de estudios superiores e incrementan las oportunidades en el mercado de trabajo. A partir de entonces y hasta la última aplicación de la prueba PISA en el 2018 (OCDE, 2019), se mantiene el interés por identificar la relación entre la motivación y el rendimiento escolar.

Tanto la OCDE como la United Nations International Children's Emergency Fund (UNICEF, por sus siglas en inglés) recomiendan continuar con el estudio de la motivación escolar de manera más amplia, considerando la participación de los docentes, los padres y el contexto, además de hacerlo de forma temprana en el 
desarrollo infantil, preferentemente desde el inicio de la primaria (OCDE, 2016).

De manera general la motivación escolar es considerada como un factor psicológico que se relaciona con: el éxito y fracaso de los aprendizajes en alumnos de preescolar, primaria y secundaria (AGUERRONDO; VAILLANT, 2005; SÁNCHEZ; LÓPEZ, 2005); con la deserción en el nivel medio superior (MIRANDA, 2018) y con la permanencia o eficiencia terminal de los estudiantes universitarios (VELÁZQUEZ; GONZÁLEZ, 2017). De forma específica dicha relación requiere de mayor atención para identificar qué motiva o no a los alumnos hacia la obtención de conocimientos

Sin duda otro factor que anima al estudio de los motivos hacia el aprendizaje es la evidencia empírica de padres, profesores, pedagogos y psicólogos educativos, sobre la necesidad de conocer las causas del bajo rendimiento escolar en niños de educación básica que no se explican por déficits cognitivos, conflictos emocionales personales y familiares o con problemas en las interacciones sociales, ya sea con iguales o autoridades.

Finalmente, la importancia del estudio de la motivación escolar es la perdida de la misma, es decir la desmotivación. No nos referimos a la concepción general sobre la desmotivación que se entiende como "la falta de interés o implicación en los procesos de enseñanza-aprendizaje” (HERRERA, 2017, p.5), sino a la debilitación o desaparición del interés por aprender después de haber estado presente.

En la revisión de los estudios sobre la motivación escolar, realizados a partir de diferentes aproximaciones psicológicas, se evidenció el predominio de la concepción de la motivación como un aspecto afectivo-emocional, que de acuerdo con Enkvist (2018), han generado que los alumnos aspiren a asistir a la escuela para entretenerse, dejando de lado el interés por aprender. En coincidencia con la autora consideramos que es insuficiente para el desarrollo del interés por el conocimiento limitarse a presentar contextos agradables, materiales vistosos, situaciones divertidas y actividades lúdicas.

Una concepción diferente sobre los motivos relacionados con el aprendizaje (motivación escolar) la ofrece la aproximación psicológica 
histórico cultural, creada por L. S. Vigotsky quien estableció la estrecha relación entre el desarrollo psíquico y las diferentes actividades que el hombre realiza, premisa que retoma A.N. Leontiev para la conformación de la teoría de la actividad, que posteriormente Talizina aplica en el ámbito pedagógico. Como resultado de estos trabajos se conformó la Teoría de la actividad aplicada a la enseñanza considerada como una actividad conjunta, que posee una estructura en la cual los tipos de motivos son fundamentales para comprender el éxito o fracaso de la interiorización del conocimiento.

En base a lo mencionado, presentamos la propuesta sobre la concepción sobre sobre la motivación escolar en niños de primaria desde la aproximación de la psicología pedagógica histórico-cultural y la teoría de la actividad aplicada a la enseñanza, desde esta perspectiva se estudian los motivos hacia el aprendizaje a partir de las siguientes preguntas ¿qué es la motivación escolar? ¿cómo se forma? ¿quienes participan en su formación? y ¿cómo podemos analizarla en los alumnos?

\section{La aproximación psicológica histórico-cultural y la teoría de la actividad.}

Hacia los años 20' del siglo XX, surge el enfoque histórico-cultural como producto de los trabajos del eminente psicólogo L. S. Vigotsky quien ofreció a la psicología un nuevo legado teórico y metodológico que le ha valido el reconocimiento mundial por sus aportaciones a la redefinición del objeto de estudio de la psicología. Durante el célebre Segundo Congreso de Psiconeurología en 1924, realizado en Lenigrado, A.R., Luria conoció a Vigotsky e impresionado por sus novedosas aportaciones decidió invitarlo a colaborar en el Instituto de Psicología de Moscú, ahí, junto con A,N. Leontiev conformaron un pequeño grupo que daría origen al desarrollo la psicología y la neuropsicología histórico-cultural (LURIA, 1979).

\subsection{De la teoría histórico-cultural a la teoría de la actividad}

Vigotsky trabajó intensamente en la reconstrucción de la psicología clásica que predominaba en su época cuestionando los intentos de la 
reflexología, que reducía a respuestas reflejas la explicación de la compleja conducta humana. Los esfuerzos de Vigotsky por comunicar su tesis sobre su concepción de los procesos psíquicos se concretaron en el manuscrito El sentido histórico de la crisis de la psicología (VIGOTSKY, 1996a), documento hallado en 1959, después de la muerte de Vigotsky a los 38 años de edad (LURIA, 1979). Desde luego este autor no solo se limitó a criticar el paradigma psicológico vigente, se propuso además elaborar un modelo científico que integrara un marco teórico y metodológico congruente para el estudio de la naturaleza, génesis y desarrollo de la psique específicamente humana.

Vigotsky (1996b, 2008) estudió el desarrollo psíquico desde el punto de vista histórico basado en la filosofía marxista del materialismo dialéctico, lo cual representó analizar los fenómenos en su proceso de cambio. Dichos cambios, de acuerdo con Vigotsky, se forman a lo largo de la historia el hombre (filogenia) y de la persona (ontogenia), cabe señalar que no debe entenderse a la filogénesis ni la ontogénesis desde posición exclusivamente biológica sino social y cultural.

Vigotsky (1996b, 2008) logró conformar una base teórica para el estudio sobre el desarrollo histórico de la psique humana que parte de los siguientes principios: 1) la naturaleza de las funciones psicológicas es social y no biológica, 2) la fuente del desarrollo del hombre depende del proceso de adquisición de la experiencia cultural de la humanidad y no de las predisposiciones innatas y 3) la vía a través de la cual se adquiere la experiencia cultural es la actividad y la enseñanza organizada. A razón de estos principios Vigotsky (LURIA, 1979) decide adjetivar a su propuesta psicológica como histórico-cultural.

Los principios de la teoría histórico-cultural fueron muy bien recibidos por A.R. Luria, médico neurólogo y doctor en ciencias pedagógicas, y por A.N. Leontiev, doctor en ciencias psicológicas, quienes no dudaron en conformar el grupo de estudio sobre los procesos psíquicos bajo este nuevo paradigma.

A.R. Luria (1902-1977) realizó importantes estudios a partir del enfoque histórico-cultural en pacientes con enfermedades neurológicas o con alteraciones por daño local a causa de heridas de bala. El objetivo principal del trabajo de Luria fue la aportación de la descripción de las alteraciones de origen neurológico 
basadas en una explicación psicológica, lo cual dio origen a la neuropsicología (LURIA, 1979; SHUARE, 1990).

A.N. Leontiev (1903-1979), participó realizando el análisis de la estructura de las diferentes actividades culturales específicamente humanas, de sus motivaciones e intencionalidades y el papel de estas actividades en el desarrollo de las funciones psicológicas superiores (DAVIDOV; SHUARE, 1987; LEONTIEV, 1986, 1993, 2010).

Poco después se unieron al grupo cinco jóvenes estudiantes, A.V. Zaporozhets, L. I. Bozhovich, N.G. Morozova, L. Slavina y R. Levina y unos años más tarde D. B. Elkonin y P.Y. Galperin (LURIA, 1979, SHUARE, 1987), quienes bajo la dirección de Vigotsky realizaron diversas investigaciones dentro de nueva psicología del desarrollo y la psicología pedagógica.

Los estudios dentro de la psicología pedagógica se orientaron: al análisis de la dirección del proceso de enseñanza y las leyes psicológicas que la caracterizan; de investigar cómo se forman los procesos cognoscitivos y de las condiciones en las que se logra el desarrollo de los procesos psíquicos durante la enseñanza; de analizar las interrelaciones entre el profesor y el educando así como las que existen entre los propios educandos; también se encarga del análisis de las formas del trabajo para la enseñanza colectiva e individual cuando el alumno lo requiere (PETROVSKI, 1980).

Por su parte, la psicología del desarrollo (denominada evolutiva en los textos clásicos) estudia la ontogénesis de los procesos psíquicos, sus particularidades de acuerdo a los diferentes periodos que atraviesa: infancia, adolescencia, primera juventud, adultez y la vejez. Además, se interesó por las formas de asimilación del conocimiento y la conformación de la personalidad. Ambas ramas de la psicología permitieron la creación de la Teoría de la Actividad.

A. V. Zaporozhets (1905-1981), otro brillante colaborador, realizó trabajos sobre el desarrollo de los movimientos voluntarios en los niños prescolares. Elaboró la teoría del desarrollo de la percepción infantil y estudió junto con L. Bozhovich la formación eficaz de la personalidad del niño como resultado de la educación y la enseñanza dirigidas (DAVIDOV; SHUARE, 1987) 
L. I. Bozhovich (1908-1981), doctora en ciencias psicológicas, se interesó en el desarrollo de la psicología soviética de la personalidad y de la educación. Con la orientación de Vigotsky trabajó en investigación científica, práctica y docente en psicología del desarrollo y pedagógica, especialmente en psicología de la personalidad (LURIA, 1979, DAVIDOV; SHUARE, 1987), se dedicó durante más de 50 años al análisis de la personalidad del escolar en la vida real (cotidiana) y en la actividad colectiva escolar. Su tesis doctoral la dedicó al proceso de asimilación de la ortografía y demostró el gran papel de los motivos en la actividad del escolar. En las investigaciones de Bozhovich sobre la psicología de la personalidad y psicología infantil, instauró un principio rector que establece que es posible comprender las leyes del desarrollo psíquico del niño solo si se considera su esfera motivacional y de las necesidades (DAVIDOV; SHUARE, 1987).

N. G. Mozorova y L. S. Slavina ambas psicólogas fueron colaboradoras cercanas de L. I. Bozhovich con quien realizaron estudios psicológicos de la personalidad en la infancia. Se interesaron por el estudio de la formación de los intereses y motivos de la actividad de estudio. Sus investigaciones se orientaron a la formación, desarrollo y transformación de la esfera motivacional en las edades, preescolar, escolar y adolescente (LEONTIEV, 1986; SLAVINA, 1985).

La pedagoga L.S. Slavina (1960) orientó su investigación hacia la actividad intelectual en niños atrasados (académicamente) y con problemas de conducta (indisciplinados), destacan en sus obras las particularidades del trabajo cognitivo de los escolares con problemas de aprendizaje, las condiciones psicológicas para elevar la actividad intelectual de los alumnos de primer grado y el desarrollo de los motivos durante la actividad de juego (ILIASOV; LIAUDIS, 1986).

D. B. Elkonin (2009) doctor en ciencias psicológica, con amplia experiencia docente en diferentes grados educativos, realizó su trabajo de investigación, bajo la dirección de Vigotsky, hacia la comprensión del desarrollo psicológico en la edad preescolar, escolar inicial, media y adolescente. A Elkonin debemos la formulación de la teoría de la periodización del desarrollo psíquico por etapas, la cual elaboró a partir de los primeros hallazgos de Vigotsky (2008), quien señaló que cada edad psicológica tiene características particulares. El 
autor también participó en la investigación sobre el desarrollo de la personalidad del niño, la formación del pensamiento, del lenguaje y el aprendizaje de la lectoescritura. Elkonin realizó relevantes aportaciones teóricas sobre los diferentes tipos de actividad infantil, entre ellas la de juego en las distintas edades infantiles. El autor junto con Leontiev elaboró el concepto de actividad rectora y establecieron la línea para su estudio en las diferentes etapas del desarrollo psicológico (DAVIDOV; SHUARE, 1987).

P. Ya. Galperin, doctor en ciencias psicológicas, trabajo muchos años como miembro del grupo de Bozhovich, Z A. Zaporozhets y Zinchenko, juntos iniciaron un nuevo ciclo de trabajos de investigación teórica y experimental estableciendo de nuevas vías metodológicas. La importancia fundamental de la teoría de Galperin (2009) consiste en que fue creada una nueva vía para estudiar la naturaleza y las leyes de formación y funcionamiento de la actividad psíquica. Galperin formuló la teoría de la formación de las acciones mentales por etapas, dicho de otro modo, sobre cómo se puede lograr la interiorización de las acciones externas (materiales). Este autor señaló que solo en el proceso de interiorización, de $\mathrm{su}$ formación dirigida, puede entenderse la actividad psíquica. Este principio de la "interiorización dirigida" adquirió el estatus de método de investigación psicológica y pedagógica gracias a la nueva visión metodológica propuesta por Vigotsky, que consistía en analizar los procesos en lugar de los fenómenos, lo cual implica conocer el origen y las etapas de la formación de dichos procesos. Evidentemente Vigotsky tuvo que justificar el porqué de su propuesta y cómo lo haría, lo cual hizo a partir de sus trabajos sobre la relación entre aprendizaje y desarrollo.

Vigotsky (2008) en sus experimentos con tareas de tipo intelectual identificó que los niños que realizaron correctamente, y de manera autónoma su primer tarea de evaluación, tuvieron un mejor desempeño en las subsecuentes tareas del mismo tipo, sin embargo, aquellos niños, que de forma autónoma no lograron realizar exitosamente las tareas por sí mismo la primera vez, y posteriormente se les otorgó ayudas para realizarlas, también evidenciaron mejor desempeño, incluso mayor al de los niños que no recibieron apoyos. Este 
fenómeno llevó a Vigotsky a establecer una de sus tesis fundamentales para el ámbito educativo, el aprendizaje es lo que conduce al desarrollo y no viceversa (VIGOTSKY, 2008), contradiciendo la tan generalizada y aún presente idea en los modelos constructivistas que señala que una buena enseñanza debe basarse en el desarrollo adquirido por el niño (OBUJOVA, 1996).

Para comprender la relación entre aprendizaje y desarrollo, Vigotsky (2008) planteó la necesidad de identificar cuando menos dos niveles del desarrollo del niño para establecer, en cada caso particular, la capacidad de aprendizaje. Estos niveles los denominó: 1) nivel o zona de desarrollo actual o real (lo adquirido) que se valora a partir de lo que logra realizar el niño de manera independiente, y 2) el nivel o zona de desarrollo potencial (próximo), que es aquello que el niño puede lograr con la ayuda de otros, principalmente de los adultos. Vigotsky señaló que trabajar con los niños en la zona de desarrollo próximo permite saber cómo están ocurriendo los procesos, como se están desarrollando y con ello establecer los objetivos para organizar o reorganizar el desarrollo, en caso de alteraciones.

[...] el aprendizaje no es en sí mismo desarrollo, pero una correcta organización del aprendizaje del niño lleva al desarrollo mental, activa todo un grupo de procesos de desarrollo, y esta activación no podría producirse sin el aprendizaje. Por ello, el aprendizaje es un momento intrínsecamente necesario y universal para que se desarrollen en el niño esas características humanas no naturales, sino formadas históricamente. (VIGOTSKY, 2008, p.37).

El interés de Vigotsky por el estudio del proceso de desarrollo psicológico ontogenético lo lleva a proponer el análisis de la siguiente forma: a) estudiar los fenómenos psíquicos es su proceso y no como objetos (fijos y estables), b) explicar las relaciones dinámico-causales de los fenómenos (aspectos internos) y no solo la descripción de los aspectos observables (externos), y c) estudiar de manera evolutiva (histórica) el proceso de cambios cualitativos del desarrollo. A este método de análisis Vigotsky (2008) lo llamó “experimental-evolutivo" (p. 100) o "causal-genético" (DAVIDOV; SHUARE, 1987; SHUARE, 1990), DAVIDOV (1988) lo denominó genético modelador (p. 326) y Talizina (2009) “experimento 
formativo" (p. 28), en el sentido de que se recrea artificialmente un proceso de desarrollo psicológico lo cual permite el estudio de los procesos en su dinámica. La participación del adulto en este método es fundamental.

Vigotsky realizó investigaciones a través del método de constatación, pero solo como forma exploratoria complementaria, la razón es que dicho método solo permite determinar el nivel de dominio de la acción o actividad, que tiene cada participante de la investigación y no su proceso de formación (SHUARE, 1990). Es decir, permite la valoración de los procesos en su forma actual y no potencial. Por el contrario, el experimento (método) formativo, es posible investigar el proceso por el cual surgen las nuevas estructuras psíquicas por medio de la formación dirigida (VIGOTSKY, 1996a, 2008).

\subsection{El surgimiento de una nueva categoría psicológica: la Actividad}

La investigación de la génesis de cualquier función psíquica, de acuerdo con Vigotsky, solo es posible a través de la reproducción sistemática, y únicamente se puede realizar con la intervención activa del investigador en los distintos tipos de actividad. De aquí la relevancia de estudiar las funciones psíquicas en las diferentes actividades humanas, tanto de su estructura -en la cual los motivos constituyen un elemento fundamental- como de su función (LEONTIEV, 1979, 1993, 2009; VIGOTSKY, 1996b, 2008).

Leontiev (1903-1979) desarrollo "la idea fundamental de la actividad como objeto de estudio" (DAVIDOV; SHUARE, 1987, p. 10) que inicialmente planteó Vigotsky. La propuesta esencial del autor fue el estudio de la psique a partir de la actividad que éste realiza, la cual describe, de manera general, como un sistema que tiene estructura (motivo, acciones y operaciones), transiciones y transformaciones, tiene su propio desarrollo y puede ser externa e interna (LEONTIEV, 1979, 1986, 1993, 2009). Leontiev puso particular empeño en el desarrollo de concepto psicológico de actividad, enfatizó la importancia de entenderla como una unidad molecular específica, que no debe confundirse con las acciones corporales del sujeto (actividad global), no es una reacción ni un conjunto de reacciones, no se refiere a la conducta general, tampoco son actos 
reflejos u operaciones aisladas como la respiración, ya que para estos actos no existe un motivo específico para su realización. Dichos actos son incluidos como fondo de actividad durante su realización y pueden ser de tipo motor (TALIZINA; SOLOVIEVA; QUINTANAR, 2010).

La actividad específica es un proceso desplegado en el tiempo, realizado por un sujeto y sometida a un motivo (SOLOVIEVA, 2009; TALIZINA; SOLOVIEVA; QUINTANAR, 2010), esto significa, desde el punto de vista psicológico, que existe un motivo el cual determina los procesos que constituyen la actividad. Algunos ejemplos de actividades específicas son; actividad de juego, actividad de creación literaria, actividad interpretativa (de una obra musical) o actividad de aprendizaje.

Leontiev señaló que la actividad específica del "ser humano es un sistema subordinado a un sistema de relaciones sociales, de tal forma que fuera de estas relaciones humanas, la actividad no tiene existencia alguna" (2010, p.18). Dichas actividades se relacionan con las necesidades determinadas por las condiciones de vida: por el lugar donde se vive y el lugar que el sujeto ocupa en el sistema de relaciones sociales.

Cualquier actividad humana está dirigida a satisfacer necesidades y su rasgo principal es que ésta tiene un objetivo (LEONTIEV, 1979), tal objetivo, que se refleja psíquicamente en forma de imágenes o representaciones, pensamientos o conceptos, es lo que estimula y dirige a la actividad en búsqueda de satisfacer una necesidad determinada. Es decir, el reflejo psíquico del objetivo se llama motivo (LEONTIEV, 1960).

Las investigaciones de Leontiev le permitieron establecer las características generales de las necesidades, la primera, es que toda necesidad tiene un objeto cultural que la puede satisfacer. Si la necesidad se cubre con "un objeto", entonces hablaremos de necesidades objetivadas. El encuentro de la necesidad con su objeto implica aparición de un motivo (representación interna del objeto) concreto de una actividad. La segunda característica señala que toda necesidad adquiere un contenido concreto según las condiciones y la manera como se satisface, ello depende del contexto. La tercera característica establece que una 
misma necesidad puede repetirse, ello en relación a las nuevas o mismas actividades. Finalmente, la cuarta característica es que las necesidades se desarrollan a medida que se amplía el círculo de objetos y de medios para satisfacerlas (LEONTIEV, 1960).

A razón de los aspectos generales expuestos sobre la teoría de la actividad se puede entender que el proceso de aprendizaje se considera como actividad, por lo tanto, la Teoría de la actividad aplicada a la enseñanza estudia la actividad de aprendizaje. Como cualquier otra actividad, la actividad de aprendizaje tiene una estructura general que incluye la realización de diferentes tipos de acciones cognoscitivas; lectura, escritura, cálculo, y en cada una de ellas debe conocerse su estructura, componentes y partes funcionales, sus características básicas, las etapas y las regularidades de su formación (LEONTIEV, 1993; TALIZINA, 1998, 2009). De acuerdo con la teoría de desarrollo psicológico por etapas desarrollada por Elkonin $(1986,2011)$ la actividad de aprendizaje es la actividad rectora de la etapa escolar.

La actividad rectora, es un aspecto característico de las edades psicológicas, es una categoría conceptual desarrollada por A.N. Leontiev y D.B. Elkonin que consigna a la actividad predominante en cada etapa del desarrollo. Dicha actividad tiene particular importancia para el desarrollo del niño, pues es en ella donde se abren las mayores posibilidades de desarrollo, esto no significa que al mismo tiempo se lleve a cabo el desarrollo en otras actividades (ELKONIN, 2011).

Elkonin propuso un tipo de actividad rectora en cada etapa a partir de un orden lógico en correspondencia a la alternancia del tipo de relaciones del niño (línea de desarrollo) y a los motivos de dicha actividad en cada edad. El autor refiere que en la edad escolar básica (primaria), la actividad rectora es la actividad de estudio y el motivo de ésta es la adquisición de aprendizajes y conceptos científicos (ELKONIN,1986). 


\section{El estudio de motivos desde la teoría de la actividad y su relación con la actividad de aprendizaje}

La motivación escolar, basada en la teoría de la actividad, se denomina como "esfera de los motivos relacionados con el aprendizaje", la cual es parte de la esfera general de los intereses y motivos de la personalidad. Como ya se mencionó, los motivos son un componente estructural de las diferentes actividades, por lo tanto, también es el primer eslabón para el proceso de asimilación de las actividades cognoscitivas. El estudio de la conformación de la esfera de motivos hacia el aprendizaje debe realizarse dentro de la actividad escolar (TALIZINA, 1998, 2009).

En la etapa escolar la formación de cualquier conocimiento y de cualquier acción, depende, antes que nada, del deseo de los alumnos para obtenerlos, por ello desde la teoría de la actividad, el problema de la formación de los motivos es esencial (LEONTIEV, 1993, TALIZINA, 2009), lo cual representa formar en los niños la necesidad de saber, de obtener conocimientos.

En la etapa escolar básica (primaria) se establecen diversos tipos de necesidades de acuerdo con el contexto, que no necesariamente corresponden a la actividad rectora de la edad psicológica, que es la actividad cognoscitiva (LEONTIEV, 1960, 1986). Esto sucede porque no existe una preparación psicológica para que el niño ingrese a la primaria, donde se espera que adquiera los conocimientos formales (científicos) (DAVIDOV, 1988; LEONTIEV, 2010, TALIZINA, 2009).

\subsection{La preparación para el ingreso a la etapa escolar.}

En la teoría de la actividad aplicada a la enseñanza, la teoría sobre la periodización del desarrollo y la formación de las acciones mentales por etapas, se considera especialmente importante la preparación de los niños para la escuela. N.G Salmina (2010) señala que "la preparación para el ingreso a la etapa escolar es uno de los componentes básicos que garantizan la adaptación al colegio y los éxitos del aprendizaje escolar" (p.67). Esta formación compleja incluye, además de los aspectos biológicos (morfo-funcionales, como el desarrollo del habla y oído 
fonemático), determinado nivel de dominio de los conocimientos específicos objetales y la preparación psicológica.

La preparación para el inicio de la etapa escolar requiere conocer las características de la actividad rectora, que en el caso de la etapa escolar es la actividad de estudio (aprendizaje). M.I. Lisina (2010), dice que analizar cualquier tipo de actividad significa identificar su objeto, cuáles son las necesidades y motivos que lo impulsan, y describir los tipos de acciones y operaciones que conforman esa actividad. Estas afirmaciones permiten entender el fracaso escolar como el resultado de la inadecuada organización de la enseñanza, por la ausencia del establecimiento de objetivos cognitivos y la formación del motivo, el deseo de aprender.

El ingreso a la etapa escolar, y en algunos casos desde la etapa preescolar (privada), los niños experimentan nuevas y diferentes exigencias ante el reto de iniciar los aprendizajes formales (científico). Los datos empíricos sugieren que a los alumnos se les requiere frecuentemente que tenga un comportamiento bajo nuevas normas estrictas, por ejemplo, la limitación de la movilidad: no puede levantarse y salir, cuando lo desee o lo necesite; no puede preguntar cuando tiene alguna duda, se le pide permanentemente que este en silencio, no puede voltear hacia su compañero para solicitar materiales o apoyo etc.

Cuando los cambios son drásticos y desagradables de un nivel escolar a otro, en el alumno se forman estados de desagrado, apatía y miedo relacionados con la escuela, el maestro y los aprendizajes, el niño no comprende el porqué de este cambio, no lo entiende porque no fue preparado. El encuentro con la actividad escolar en estas condiciones no les genera ninguna alegría a los niños, ninguna satisfacción, menos aún motivación para el aprendizaje (SALMINA, 2010). La ausencia de satisfacción aumenta aún más si las materias escolares o la forma de enseñanza no generan ningún tipo de interés, ni la participación activa por parte del alumno.

Al principio de la vida escolar el niño aún no experimenta la necesidad de conocimientos teóricos como base psicológica de la actividad de estudio (Davidov y Markova, 1986), dicha necesidad "surge en el proceso de asimilación real de los 
conocimientos teóricos elementales durante la realización conjunta con el maestro de las acciones de estudio más sencillas dirigidas a la solución de las correspondientes áreas de estudio" (p. 318).

De acuerdo con Davidov y Markova (1987) el profesor debe desarrollar la necesidad del conocimiento, crear motivos generales que inciten a la realización de la actividad de estudio con un claro sentido de lo que se hace en la escuela, aprender.

En el análisis estructural y funcional de las acciones escolares, como la lectura, la escritura y el cálculo, señala que el primer elemento lo constituye el objetivo de la acción, que le permitirá al estudiante dirigirse a él de forma consciente. La posibilidad de tener presente el objetivo, se relaciona inseparablemente de otro componente, el motivo de la acción, cuyo papel es impulsar al niño para el establecimiento y logro de los objetivos. Leontiev (1993) señala que "lo que define el éxito en el proceso de resolver la tarea no es solo el contenido objetivo, sino que en primer término depende del motivo que impulsa al niño a actuar o, dicho de otro modo, del sentido que tiene para él esa actividad” (p. 222).

Solovieva, Rosas y Quintanar (2016) aplicaron el método para enseñar el proceso de resolución problemas matemáticos en la escuela primaria basado en la teoría de la actividad y del enfoque histórico y cultural, y constataron que el programa posibilitó la aparición y conservación de la motivación positiva por las matemáticas en el grupo experimental.

\section{4. ¿Qué es la motivación escolar?}

Desde la teoría de la actividad aplicada a la enseñanza la motivación escolar se denomina "esfera de los motivos relacionados con el aprendizaje" y se constituye por los diferentes tipos de motivos que forman los sentidos, positivos o negativos hacia la actividad de estudio, es decir de los significados propios del niño hacia el aprendizaje. Dicha construcción se organiza de forma jerárquica y es dinámica. Jerárquica porque se puede identificar el predominio de un determinado tipo de motivos sobre otros y dinámica porque se puede reorganizar. 
Talizina (1998, 2009) dedicó gran parte de su trabajo a la investigación de los motivos relacionados con el aprendizaje y señaló la posibilidad de caracterizarlos de acuerdo a su contenido y al estado o nivel de su formación.

En relación con el contenido, los motivos del aprendizaje se pueden dividir en: 1) motivos escolares cognoscitivos (internos), relacionados con el proceso de aprendizaje y 2) motivos escolares generales (externos), relacionados los aspectos contextuales de la vida del niño.

De acuerdo al estado o nivel de su formación, los motivos de escolares se caracterizan por 1) por ser conscientes o inconscientes, 2) por la comprensión de importancia de éstos motivos y 3) por la medida de la fuerza de activación del motivo (TALIZINA, 2009, p. 69). Estos rasgos permiten establecer en su conjunto el nivel de desarrollo de la esfera motivacional escolar.

La propuesta de Talizina (1998, 2009), para el análisis de los motivos escolares se basa en la siguiente tipología.

1) Los motivos de tipo interno o cognoscitivo, los cuales orientan la actividad del niño hacia la obtención de saberes. Los motivos de tipo cognoscitivo se vinculan estrechamente con la actividad escolar, con el logro académico. Talizina añade que las particularidades de los motivos escolares pueden garantizar el éxito de los aprendizajes, en especial si se dirigen hacia la satisfacción de una necesidad cognoscitiva (interés por conocer), este tipo de motivos no surge intrínsecamente, como producto del desarrollo evolutivo desde el punto de vista biológico (madurez), por lo tanto, los motivos de tipo interno deben ser formados positivamente por los adultos (padres y profesores).

Cabe precisar que Talizina señala que existe un solo tipo de motivos internos, la pluralización del término "motivos" obedece a que existen tantos motivos como actividades de aprendizaje existan, cada una de ella tiene su propio motivo interno.

2) Los motivos externos (ajenos al deseo de aprender), no se relacionan con los conocimientos, ni con la actividad escolar que se realiza y pueden o no contribuir en el proceso de aprendizaje. Estos motivos se asocian a la situación escolar general, como las instalaciones, los materiales y equipos, las interacciones 
sociales (con compañeros, profesores o personal del colegio), las actividades cocurriculares, deportivas o las lúdico-recreativas.

Los motivos externos se pueden clasificar en tres subtipos: a) los positivo, b) los neutros y c) los negativos. Los motivos externos neutros no son explicitados por Talizina, pero se infiere su presencia y contenido en el análisis teórico de sus trabajos. La caracterización de los motivos externos se puede resumir de la siguiente manera:

a) Los motivos externos positivos son aquellos que apoyan la actividad de aprendizaje escolar, que hacen agradable la obtención de conocimientos, que mantienen el interés por la actividad de estudio, es decir apoyan el deseo por el conocimiento. Algunos ejemplos de los motivos externos positivos, de tipo material, se asocian a la utilización de objetos atractivos en forma color o novedosos que se usan como medios para adquirir conocimientos. Otro ejemplo de motivos externos positivos son la obtención de atención y reconocimiento de sus profesores en relación a su desempeño, las orientaciones y correcciones ante sus errores académicos a través de las actitudes amables y tolerantes. Este tipo de motivos genera en los niños la aproximación a las actividades de estudio de forma agradable.

b) Los motivos externos neutros, son aquellos que hacen confortable la estancia escolar, pero no contribuyen a la asimilación de los conocimientos. Estos motivos generan bienestar en general, comodidad, pero no relacionada directamente con el interés por el aprendizaje. Algunos ejemplos de estos motivos son las instalaciones; amplias, cómodas, higiénicas, seguras, etc, y los aspectos estéticos como el diseño y la decoración.

c) Los motivos externos negativos, tienen la característica de generar emociones o afectos desagradables, miedo, ansiedad, tristeza, disgusto hacia las actividades de estudio. Algunos de estos motivos pueden impedir que se forme el interés por el aprendizaje y peor aún, pueden destruir el motivo cognoscitivo, son la causa de la desmotivación, es decir que habiendo adquirido en etapas previas el deseo de aprender (motivo cognoscitivo) éste puede desaparecer ante la presencia de la motivación externa negativa por parte de los adultos o 
compañeros. Cabe señalar que, en el caso de los motivos negativos asociados a la obtención de beneficios, la ansiedad, miedo o desagrado surge de la presentación condicionada o coaccionada de los objetivos de las acciones escolares (aprendizajes). De esa forma, la actividad de estudio adquiere sentidos negativos en los niños. Por ejemplo, si alguien dice ¡Si no te aprendes las tablas no te dejo salir a jugar con tu primo!, isi no haces bien tu tarea le diré a tu ... que no te lleve al futbol!, isi sacas diez en el bimestre te compro...!

La presencia de motivos externos negativos se expresa como desinterés por las actividades de estudio a través de la evitación, aplazamiento, también se observa desagrado, sufrimiento y temor mientras se realiza la actividad de aprendizaje. Los motivos externos negativos incluso pueden volver verdaderamente insoportable la actividad de estudio para los niños, que se observa en la resistencia o negativismo hacia cualquier actividad de tipo académico. Por lo tanto, este tipo de motivos, generadores de malestar emocional y físico, no favorecen el aprendizaje y si lo comprometen.

\section{5. ¿Cómo se conforma la motivación escolar?}

Desde la psicología pedagógica y la teoría de la actividad la formación de la esfera de motivos relacionados con el aprendizaje se da a través de dos vías: 1) de la asimilación del sentido social del aprendizaje y 2) a través de la misma actividad del escolar, la cual debe ser interesante para el niño por una u otra razón.

En la primera vía, la asimilación del sentido social del aprendizaje, el objetivo básico del profesor es llevar hasta la conciencia del niño aquellos motivos que poseen un significado social importante, pero que actúan fuertemente (motivos externos). Como ejemplo, puede servir el deseo de obtener buenas calificaciones o intentar de complacer a los padres generando orgullo por su desempeño u obtener un premio de celebración. Sin embargo, lo más importante en ayudar a los escolares a comprender la relación objetiva entre la calificación y el nivel de asimilación de los conocimientos y acciones. De esta forma, se puede acercar gradualmente a la motivación (interna) relacionada con el deseo para obtener un nivel alto de conocimientos y habilidades. 
La segunda vía de formación de los motivos escolares se relaciona con la organización del proceso de aprendizaje. Desarrollar condiciones concretas que producen interés del alumno con la actividad escolar es una tarea que el profesor debe realizar, no deben esperar que el interés surja de manera espontánea en los niños o que aparezca a pesar de lo desagradable de la situación de aprendizaje. Por ejemplo, en el caso de las matemáticas, las formas mecánicas de resolución de las operaciones aritméticas sin la comprensión de la esencia particular de cada una de ellas generarán en el estudiante poca atracción (SOLOVIEVA, ROSAS y QUINTANAR, 2016). La repetición de una actividad de forma indiscriminada generará agotamiento y el consecuente rechazo al tema en cuestión. De acuerdo con Talizina (2009) "los intereses cognitivos del escolar dependen, en gran parte, del medio del descubrimiento del material de estudio" (p. 231).

\section{6. ¿Quienes participan en la conformación de la esfera de motivos escolares?}

De acuerdo con la teoría de la actividad aplicada a la enseñanza el proceso de asimilación exitoso de los conocimientos requiere del paso gradual por todas las etapas (estructurales) necesarias. Dicho proceso incluye etapas básicas (TALIZINA, 1992, 2009): 1) la motivacional, 2) la elaboración de la base orientadora, 3) la realización de la actividad.

1) En la etapa motivacional el maestro debe ocuparse (desarrollar) los motivos que garantizan la aceptación por los alumnos de los conocimientos y habilidades que propone. "Cada maestro sabe qué, si el alumno no quiere estudiar, no es posible que aprenda. Esto significa que en cada escolar debe haber un motivo para estudiar" (TALIZINA, 1998, p. 78).

2) La base orientadora de la acción es el sistema de condiciones, en el que se apoya el sujeto para la realización de la acción. Las condiciones de cada acción pueden incluir las características particulares de un caso concreto, pero también pueden establecer las condiciones generales para toda una clase de fenómenos. De acuerdo con Galperin (2009) el tipo de base orientadora se relaciona con el éxito o fracaso de las acciones escolares. 
3) La realización de la actividad solo es posible a través de sus componentes, las acciones orientación, de ejecución y de control, y corrección. La parte central de la acción es su función orientadora (explicativa) la cual garantiza el éxito de la acción. En etapa funcional de ejecución ocurre la transformación del objeto de la acción dirigida a la obtención del resultado. La función de control se dirige hacia la verificación de los resultados, tanto de la parte de orientación, como de ejecución, dirigida al seguimiento de los pasos de la ejecución y la verificación de su correspondencia con el plan elaborado. La función de corrección se encarga de descubrir los errores o cambio de las formas adecuadas.

El planteamiento de las etapas generales del proceso de aprendizaje mencionadas, se sustenta teóricamente a partir de lo que Vigotsky (2008, 2012) expuso sobre el hecho de que las funciones aparecen en escena dos veces, primero de manera extrapsíquica y después como proceso intrapsíquico, así, el motivo que inicialmente fue introducido por el adulto, después será interiorizado por el niño. Solo el maestro interesado en el objeto propio de su actividad (enseñanza de una materia escolar), puede transferir este objeto (motivo) al alumno, para que este más tarde, pueda interiorizarlo (TALIZINA, 2009) y convertirlo en su propio motivo.

\section{7. ¿Cómo podemos analizar cualitativamente la esfera de motivos hacia el aprendizaje?}

En relación al diagnóstico de la motivación escolar, Talízina (1998, 2009) ha propuesto algunos métodos para identificar los niveles de motivación, entre los cuales se encuentran 1) la observación, 2) la entrevista, y 3) la creación de situaciones de elección.

A partir de a revisión teórica presentamos algunos indicadores para valorar el nivel motivacional a través de los métodos propuestos por Talizina:

1) Método de observación. Se analizan las actitudes hacia las actividades y situaciones escolares, por ejemplo, la ausencia del niño a la escuela, salidas frecuentes del salón de clase por distintas causas, escasa participación, rechazo o 
evitación para realizar actividades dentro del aula, trabajo realizado con poco esmero, incumplimiento de las tareas en casa.

2) Entrevista con el alumno. Se elaboran las preguntas relacionadas con diferentes aspectos escolares, el inmueble, los materiales y equipo, las interacciones con iguales y autoridades, las actividades de aprendizaje (materias), los procesos de evaluación, sobre las tareas en el aula y para casa. Se indaga sobre si dichas tareas o situaciones les resultaron interesantes o les parecieron difíciles, desagradables o aburridas etc. Solovieva y Mata (2019) propusieron entrevistas semiestructurada para realizar el análisis cualitativo de los motivos relacionados con el aprendizaje en niños, padres y profesores.

3) Creación de situaciones de elección. Se proponen al niño tareas lejanas a las actividades de aprendizaje (como las de ser asistente de alguien en alguna tarea propuesta, o realizar una actividad de mensajería, etc). Esta situación permite identificar si el alumno prefiere evitar las actividades de aprendizaje haciendo otro tipo de actividades. Otra variante de la situación de elección es proponer al alumno elaborar los horarios de las actividades para la siguiente semana, el niño deberá elegir aquellas que más le gustan y organizarlas en el calendario, esto permitirá identificar la jerarquía de los intereses por las diferentes actividades y establecer el nivel de motivación hacia las actividades escolares (TALÍZINA, 1998, 2009).

4) El dibujo dialogado demostró su utilidad en la investigación sobre los motivos de aprendizaje en niños de primaria. La estrategia consiste en solicitarle al niño que dibuje lo que le agrada de asistir a la escuela y los que le gustaría modificar de ella -lo que le desagrada- con el objetivo de facilitar el diálogo que permita obtener información sobre los aspectos escolares (Solovieva y Mata, 2019).

\section{Consideraciones finales}

El estudio de la motivación escolar desde la aproximación historicocultural ofrece una visión que relaciona los aspectos psicológicos y pedagógicos que ocurren en el proceso de enseñanza aprendizaje. Rebasa las 
concepciones que se limitan la explicar la motivación escolar como solo un aspecto afectivo emocional asociado.

Los interesados en el tema de los motivos relacionados con el aprendizaje tienen ahora la posibilidad de estudiarlos desde una nueva propuesta, posibilitando la generación de conocimientos en relación a la conformación de la esfera de motivos asociados al aprendizaje.

\section{Referências}

AGUERRONDO, I. Y VAILLANT, D. El aprendizaje bajo la lupa: Nuevas perspectivas para América Latina y el Caribe. Nicaragua: Fondo de las Naciones Unidas para la Infancia (UNICEF), 2005.

DAVIDOV, A; MARKOVA, A La concepción de la actividad de estudio de los escolares. En Iliasov, I; Liaudis, V (Org.). La psicología evolutiva y pedagógica de la URSS. Antología. URSS: Progreso, 1986. Pág. 316-337.

DAVIDOV, A; MARKOVA, A. El desarrollo del pensamiento en la edad escolar. En: DAVIDOV, V; SHUARE, M (Org.). La psicología evolutiva y pedagógica de la URSS. Antología. Moscú: Editorial Progreso, 1987. Pág. 173-193.

DAVIDOV, V; SHUARE, M (Org.). La psicología evolutiva y pedagógica de la URSS. Antología. Moscú: Editorial Progreso, 1987. Pág. 250-273.

DAVIDOV, V.V. La enseñanza escolar y el desarrollo psíquico. Investigación psicológica teórica y experimental. Moscú: Editorial Progreso, 1988.

ENKVIST, I. La nueva pedagogía es un error. Parece que se va a la escuela a hacer actividades, no a trabajar y estudiar. Entrevista concedida a Cristina Galindo. EL PAÍS. https://elpais.com/elpais/2018/07/17/eps/1531826084 917865.html (2018).

ELKONIN, D. Acerca del problema de la periodización del desarrollo psíquico en la edad infantil. En: ILIASOV, I; LIAUDIS, Y (Org.). Antología de la psicología pedagógica y de las edades. Cuba: Pueblo y educación, 1986. Pág. 34-40.

ELKONIN, D. Hacia el problema de periodización del desarrollo en la edad infantil. En QUINTANAR, L; SOLOVIEVA, Y (Org.). Las funciones psicológicas en el desarrollo del niño. México: Trillas, 2011. Pág. 191-209.

GALPERIN, P. La formación de los conceptos y las acciones mentales. En QUINTANAR, L; SOLOVIEVA, Y (Org.). Las funciones psicológicas en el desarrollo del niño. México: Trillas, 2009. Pág. 80-90. 
HERRERA, N. La motivación y la desmotivación en las aulas de primaria. Tesis de Maestria. 2017. Recuperado en https://riull.ull.es/xmlui/bitstream/handle/915/6497/La\%20motivacion\%20y\%20desm otivacion $\% 20 \mathrm{en} \% 20 \mathrm{las} \% 20$ aulas $\% 20 \mathrm{de} \% 20$ primaria.pdf? sequence=1\&isAllowed=y.

ILIASOV, I; LIAUDIS, Y Antología de la psicología pedagógica y de las edades. La Habana: Pueblo y educación, 1986.

LEONTIEV, A. La actividad y la personalidad. En: LEONTIEV, A (Org.). La Actividad en Psicología. La Habana: Ministerio de Educación, 1979.

LEONTIEV, A. Sobre la teoría del desarrollo de la psique del niño. En: ILIASOV, I; LIAUDIS, Y (Org.). Antología de la psicología pedagógica y de las edades.

Cuba: Pueblo y educación, 1986. pp. 10-12.

LEONTIEV, A.N. Actividad, Conciencia y Personalidad. México: Cartago de México, 1993.

LEONTIEV, A. La importancia del concepto de actividad objetal para la psicología. En: QUINTANAR, L; SOLOVIEVA, Y (Org.). Las funciones psicológicas en el desarrollo del niño. México: Trillas, 2009. pp. 54-63.

LEONTIEV, A.N. El desarrollo del niño en la edad preescolar. En: SOLOVIEVA, Y; QUINTANAR, L (Org.). Antología del desarrollo psicológico del niño en edad preescolar. México: Editorial Trillas, 2010. pp. 17-26.

LEONTIEV, AN. Las necesidades y los motivos de la actividad En: SMIRNOV, A; RUBINSTEIN, S; LEONTIEV, A; TIEPLOV, B (Org.). Psicología. México:

Editorial Grijalbo, 1960. pp. 341-354.

LISINA, M. Problemas y objetivos del estudio de la comunicación de los preescolares con sus coetáneos. En: SOLOVIEVA, Y; QUINTANAR, L (Org.). Antología del desarrollo psicológico del niño en edad preescolar. México: Editorial Trillas, 2010. Pág. 87-95.

LURIA, A.R. Mirando hacia atrás. España: Norma, 1979.

MIRANDA, L. Abandono escolar en educación media superior: conocimiento y aportaciones de política pública. SINÉCTICA: Revista Electrónica de Educación. 1-5, 2018. DOI: https://doi.org/10.31391/s2007-7033(2018)0051-010.

OBUJOVA, L. F. El aprendizaje, motor del desarrollo. Perspectivas, 26 (1), 93105. 1996.

ORGANIZACIÓN PARA LA COOPERACIÓN Y EL DESARROLLO ECONÓMICOS PISA 2018 results. What school life means for students 'lives. Volume III. 2019. 


\section{ORGANIZACIÓN PARA LA COOPERACIÓN Y EL DESARROLLO}

ECONÓMICOS. Primeros resultados del programa internacional de evaluación del programa internacional de evaluación de estudiantes (PISA) 2000 de la OCDE. 2002.

http://archivos.agenciaeducacion.cl/Reporte_internacional_version_espanol2000.pdf.

\section{ORGANIZATION FOR ECONOMIC CO-OPERATION AND DEVELOPMENT Low-Performing Students: Why They Fall Behind and How to Help Them Succeed, PISA. 2016. https://www.oecd-ilibrary.org/docserver/9789264250246- en.pdfexpires $=1572608913 \& i d=i d \& a c c n a m e=$ guest\&checksum=1ACCF8E49F8C B1BAF8CE832C6A442098.}

PETROVSKI, A. Psicología evolutiva y pedagógica. Moscú: Progreso. 1980.

SALMINA, N. Indicadores de la preparación de los niños para la escuela. En: SOLOVIEVA, Y; QUINTANAR, L (Org.). Antología del desarrollo psicológico del niño en edad preescolar. México: Editorial Trillas, 2010. pp. 67-74.

SÁNCHEZ, M; LÓPEZ, M. De la motivación y del síndrome de fracaso. México: Universidad Autonoma de la Ciudad de México, 2005.

SHUARE, M. La psicología soviética tal como yo la veo. Moscú: Editorial Progreso, 1990.

SLAVINA, L.S. Niños atrasados e indisciplinados. México: Ediciones Roca, 1985.

SOLOVIEVA, Y.; MATA, A. El dibujo dialogado para evaluación de esfera motivacional escolar en alumnos de primaria. En GONÇALVES M.; SANCHES N.; PROENÇA, M. (Eds.). Avaliação psicológica e escolarização: contribuições da psicologia histórico-cultural (pp. 339-361). Brasil: Editora da Universidade Federal do Piauí - EDUFPI. ISBN: 978-85-509-0517-4. 2019.

SOLOVIEVA, Y., ROSAS, Y.; QUINTANAR, L. Program for solving problems as method for development of logic thinking in school children. RIPEM V.6, N.2, 2016.

TALIZINA, F.N. La teoría de la actividad aplicada a la enseñanza. México: Benemérita Universidad Autónoma de Puebla, 2009.

TALIZINA, F.N. Qué enseñar y cómo enseñar hoy: Libro para el maestro. México: Atenas, 1998.

TALIZINA, N; SOLOVIEVA, Y; QUINTANAR, L. La aproximación de la actividad en psicología y su relación con el enfoque histórico-cultural de L. S. Vigotsky. Novedades Educativas. n. 230, pp. 4-8, febrero. 2010. 
VELÁZQUEZ, Y; GONZÁLEZ, M. Factores asociados a la permanencia de estudiantes universitarios: caso UAMM-UAT. Revista de la Educación Superior 46(184) 117-138. 2017. DOI: https://doi.org/10.1016/j.resu.2017.11.003.

VIGOTSKY, L.S. El desarrollo de los procesos psicológicos superiores. Barcelona: Critica, 2008.

VIGOTSKY, L.S. Obras escogidas. Tomo I. Problemas teóricos y metodológicos de la psicología. España: Visor, 1996a.

VIGOTSKY, L.S. Obras escogidas. Tomo III. Problemas del desarrollo de la psique. España: Visor, 1996b. 